\title{
Control of Solar Energy Systems *
}

\author{
Eduardo F. Camacho* Manuel Berenguel ** \\ * Department of System Engineering and Automatic Control of the \\ Escuela Superior de Ingenieros of the University of Sevilla, Spain \\ (e-mail: efcamacho@us.es) \\ ** Department of Languages and Computation of the University of \\ Almeria, Spain(e-mail: beren@ual.es)
}

\begin{abstract}
This work deals with the main control problems found in solar power systems and the solutions proposed in literature. The paper first describes the main solar power technologies, its development status and then describes the main challenges encountered when controlling solar power systems. While in other power generating processes, the main source of energy can be manipulated, in solar energy systems, the main source of power which is solar radiation cannot be manipulated and furthermore it changes in a seasonal and on a daily base acting as a disturbance when considering it from a control point of view. Solar plants have all the characteristics needed for using industrial electronics and advanced control strategies able to cope with changing dynamics, nonlinearities and uncertainties.
\end{abstract}

Keywords: control of solar energy systems, model predictive control, control of thermo solar plants, control of parabolic troughs

\section{INTRODUCTION}

The use of renewable energy, such as solar energy, experienced a great impulse during the second half of the seventies just after the first big oil crisis. At that time economic issues were the most important factors and the interest in these types of processes decreased when the oil prices fell. There is a renewed interest in the use of renewable energies nowadays driven by the need of reducing the high environmental impact produced by the use of fossil energy systems [Camacho et al., 2012].

The most abundant, sustainable source of energy is the Sun, which provides over 150,000 terawatts of power to the Earth; about half of that energy reaches the Earth surface while the other half gets reflected to outer space by the atmosphere. Only a small fraction of the available solar energy reaching the Earth surface would be enough to satisfy the global expected energy demand. Although most renewable energies derive their energy from the Sun, by solar energy we refer to the direct use of solar radiation. One of the greatest scientific and technological opportunities we are facing is to develop efficient ways to collect, convert, store, and utilize solar energy at affordable costs [Bose, 2010].

There are two main drawbacks of solar energy systems: a) the resulting energy costs are not yet competitive and b) solar energy is not always available when needed. Considerable research efforts are being devoted to techniques which may help to overcome these drawbacks, control is one of those techniques.

\footnotetext{
* This work was supported in part by the Spanish Ministry of Science and Innovation and Junta de Andalucía under grants DPI200805818, DPI2010-21589-C05-01/04, and P07-TEP-02720).
}

While in other power generating processes, the main source of energy (the fuel) can be manipulated as it is used as the main control variable, in solar energy systems, the main source of power which is solar radiation cannot be manipulated [Camacho et al., 1997] and furthermore it changes in a seasonal and on a daily base acting as a disturbance when considering it from a control point of view. Solar plants have all the characteristics needed for using industrial electronics and advanced control strategies able to cope with changing dynamics, (nonlinearities and uncertainties). As fixed PID controllers cannot cope with some of the mentioned problems, they have to be detuned with low gain, producing sluggish responses or if they are tightly tuned they may produce high oscillations when the dynamics of the process vary, due to environmental and/or operating conditions changes. The use of more efficient control strategies resulting in better responses would increase the number of operational hours of the solar plants and thus reduce the cost per kw-h produced.

From a control point there are different control levels; a) the control of the movement of the solar collectors (sun tracking), b) the control of the thermal process variables, c) the plant wide control aspects and d) the grid integration.

The control of the movement of the collectors is usually done in an open loop fashion with some minor closed loop adjustments. The control of the thermal process variables consist of maintaining the process main variables closed to their set point which is usually done in a closed loop fashion. The determination of the the setpoints is done at the plant-wide control level. The grid integration control plans the production for different time horizons. 
This work describes the main solar energy plants and the control problems involved and how control systems can help in increasing their efficiency.

The paper is organized as follows: section 2 describes the main technologies used for solar energy production. The main control problems encountered are described in section 3. Section 4 focuses on some of the control problems found in solar towers while section 5 is devoted to parabolic trough collectors, with a real application of a model predictive control algorithm.

\section{SOLAR ENERGY HARVESTING}

Solar powered electrical generation can be done either directly, by the use of photovoltaic (PV) cells or indirectly by collecting and concentrating the solar power (CSP) to produced steam which is then used to drive a turbine to provide the electrical power.

The direct generation of electricity from solar energy is based on the photovoltaic effect which refers to the fact photons of light knock electrons into a higher state of energy. Although the first application of photovoltaics was to power spacecrafts, there are many PV power generation for everyday life applications such as grid isolated houses, pumps for water extraction, electric cars, roadside emergency telephones and remote sensing [Carrasco et al., 2006, Liserre et al., 2010].

Concentrating solar thermal (CST) systems use optical devices (usually mirrors) and Sun tracking systems to concentrate a large area of sunlight into a smaller receiving area. The concentrated solar energy is then used as a heat source for a conventional power plant. A wide range of concentrating technologies exist. The main concentrating concepts are: a) parabolic troughs, b) solar dishes, c) linear Fresnels, and d) solar power towers. The main purpose of the concentrating solar energy is to produce high temperatures and therefore high thermodynamic efficiencies.

\section{SOLAR PLANTS CONTROL CHALLENGES}

This section describes the main issues encountered when controlling thermal solar plants. The section concentrates in the solar side of the plant and not in the more conventional part. The main controls of solar plants can be classified in Sun tracking and control of the thermal variables. While the control of the Sun tracking mechanisms is typically done in an open loop mode, the control of the thermal variables is mainly done in closed loop.

A solar tracker is a device that points a solar collector mechanism towards the Sun or direct a reflector mechanisms in such a way that it reflects the maximum energy on a collector device. The solar power received by a solar collector is equal to the solar radiation received at that location multiplied by the devices surface and by the cosine of the angle form by the Sun rays and the surface normal. There are many types of solar tracking mechanisms with different accuracy. Power tower heliostat need a good degree of accuracy to ensure that the power is reflected on the receiver which can be situated hundreds of meters from the heliostat. Little accuracy is required for non-concentrating applications; in fact, most of these applications work without any solar tracking at all. Tracking can increase significantly the amount of energy produced, specially in the early morning and late afternoon when the cosine the angle of the direct solar radiation with the surface normal is smaller.

Many fast algorithms for the calculation of the solar position, used in engineering application which require small computation, can be found in the literature. There has been a number of algorithms proposed in literature [Blanco et al., 2001, Chen et al., 2006, Grena, 2008] which increase the precision without incurring in high computational efforts. These algorithms work correctly for limited periods of time. There are also high-precision astronomical algorithms to compute the Sun position with an error smaller than 0.0003 degrees on a very long period of time (2000 b.C. - 6000 a.C.) they require of a large amount of computation.

\section{SOLAR POWER TOWER CONTROLS}

A Solar Power Tower (SPT) plant consists of of the heliostat field, receiver unit, heat transfer, exchange and storage unit, steam and electricity production units and the integrated control system. Usually each of the units has its specific control device. The integrated control system communicates with the different subsystems to coordinate the different units in such a way that the plant operates in a safe and efficient way.

Typically a plant control system includes heliostats control and heliostats field dispatch optimization, water level control in receivers, main steam temperature control, steam supply pressure and temperature in heat storage system control under heat releasing condition, and the main steam pressure control. There are only two commercial tower power plants in operation, the $10 \mathrm{Mw}$ (PS10) and the 20 Mw (PS20) plants (figure 1), designed, build and operated by Abengoa Solar closed to Seville in Southern Spain [Abengoa-Solar, 2009].

The master control system of a solar power plant PS10 plant in Spain consists of different levels. The first level is Local Control, it takes care of the positioning of the heliostats when the aiming point and the time are given to the system, and informs upper level about the status of the heliostats field. The second logic level is distributed control system, which makes some important dispatch calculations of heliostats field. The PS20 consists of a solar field made up of 1,255 mirrored heliostats designed by Abengoa Solar. Each heliostat, with a surface area of 120 square meters, reflects the solar radiation it receives onto the receiver, located on the top of a 162 meters-high tower, producing steam which is converted into electricity generation by a turbine.

The Sun vector needs to be computed and for each heliostat, the normal vector is computed in such a way that it divides the angle formed by the Sun vector and the vector joining the center of the heliostat with the receiver.

The current trend in solar concentrator tracking systems is to use open-loop controllers that compute the direction of the solar vector based on location and time [Blanco et al., 2001]. When controlling the temperature and flux distribution in the receiver, the algorithms calculate the amount of the shift using an equation appropriate for each 


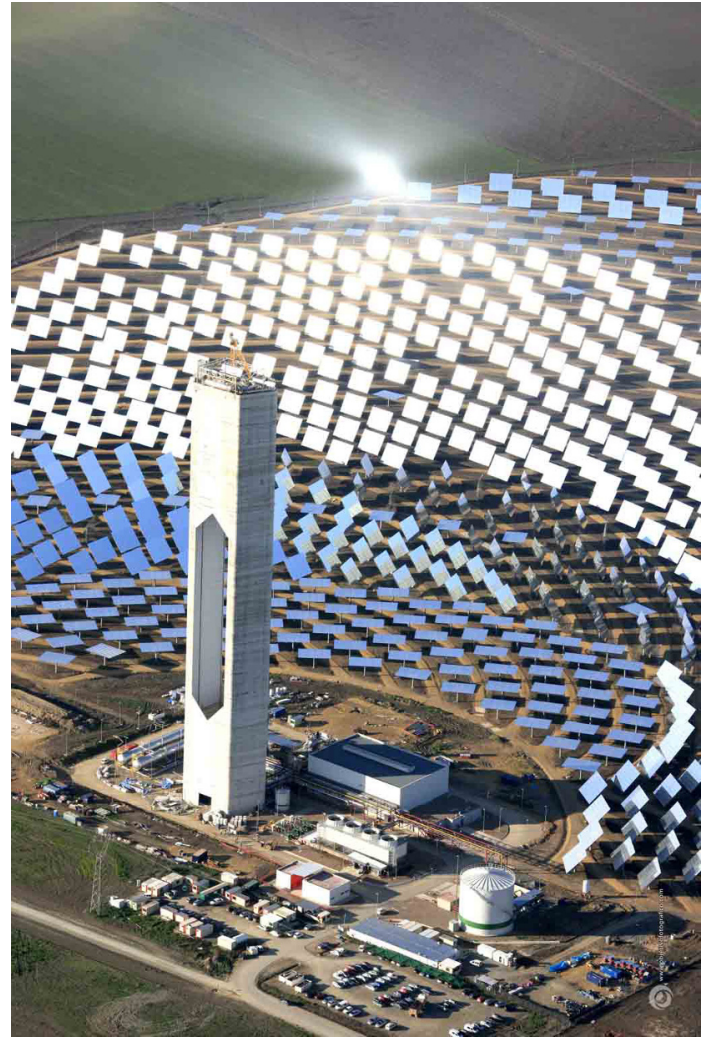

Fig. 1. Abengoa PS20: a 20Mw solar power tower

heliostat depending on its current temperature dependent focal length and orientation dependent aberrations in addition to beam errors and strategy providing a desired energy flux can be selected to solve the control problem. Nevertheless, there are error sources that increase the complexity of the control system [Stone and Lopez, 1995] such as time, Sun model, latitude and longitude of the site, heliostat position in the field, time-varying astigmatism, cosine effects, processor accuracy, atmospheric refraction, control interval and structural, mechanical and installation tolerances. Some of these error sources are systematic ones, mainly due to tolerances (joints, encoder, etc.), wrong mirrors facets alignment (optical errors), errors due to the approximations made when calculating the solar position [Blanco et al., 2001], etc. Heliostat beam quality error sources are analyzed in [King and Arvizu, 1981].

The solution proposed in [Berenguel et al., 2004] to overcome some of these error sources (mainly those related to the calculation of the solar position and to tolerances) is based on the use of a CCD camera which captures images of the Sun reflected by each of the heliostats of the field onto a target used for offset correction. The reflection of the light coming from the Sun produces a shape that continuously changes due to the Sun-Earth relative movement. The obtained images serve as feedback information that allows the automatic calculation of the distance between the center of the target and the sunbeam centroid in such a way that this error signal was used for adjustment purposes. After the calculation of the required displacement of the motors of the heliostats (encoder steps), the system sends this information to the central control system to perform the correction.

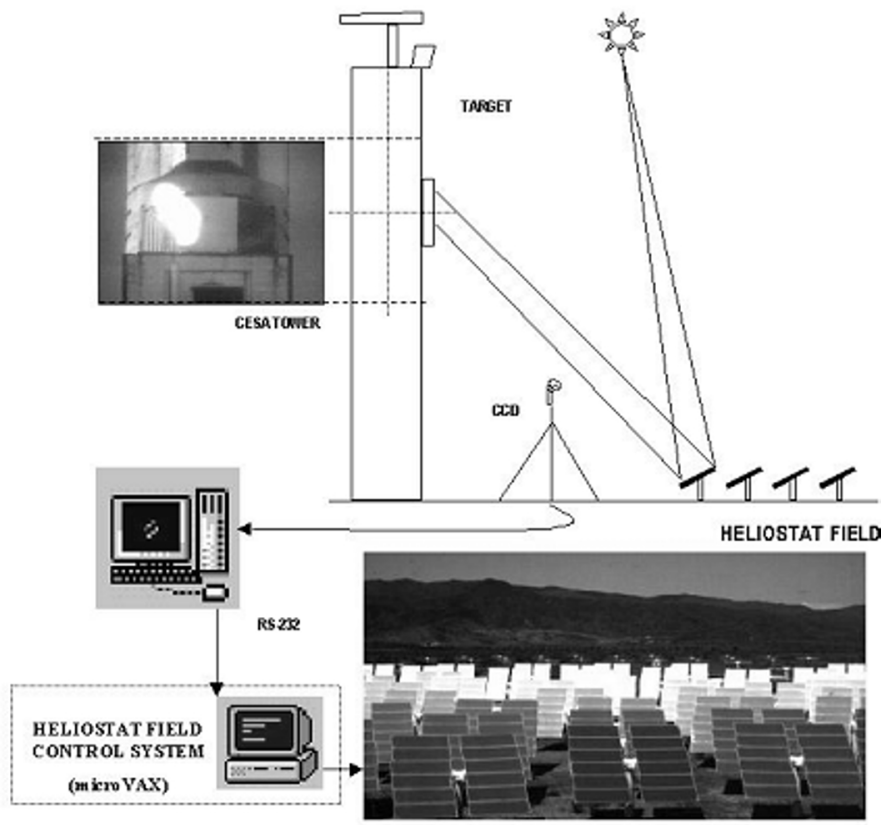

Fig. 2. Heliostat calibration

The offset correction problem consists in comparing the actual sunbeam centroid position on a target to a command reference position to determine the error in the sunbeam centroid location. The sunbeam centroid position error is then analysed to correlate the error to errors in the heliostats track alignment system. New coefficients are established for the heliostats track alignment system to automatically correct for errors in the system, this eliminates the need for aligning heliostats. The aimpoint errors are corrected by changing the database stored values. A CCD camera (equipped with a pan-tilt mechanism and with an automatic contrast/brightness adaptation mechanism) is used and the heliostats are moved until the sunbeam centroid coincides with the center of the target (figure 2). These control action are send to the local controllers of the servomotors to move the heliostats to the positions. The system can also be used during operation, as one heliostat can be deviated from its spot to correct its offset in real time.

One of the main problems in operating receivers is to obtain an appropriate flux distribution in order to avoid deterioration due to excessive thermal gradients. Concentrating solar receivers having heliostat where all heliostats are aimed at the same point leads to peaked nonuniform distribution. One way to overcome a nonuniform irradiance profile is the multi-aiming strategy, whereby the individual heliostats are deliberately aimed at different aiming points in such a way that the peaked irradiance profile due to central aiming is spread to a more uniform irradiance profile over a much larger aperture area. The number and location of the aiming points are usually determined by simulation under different irradiance conditions. Typically five aiming points, one at the receiver center and the remaining four at the periphery are enough. An heuristic algorithm was presented in [García-Martín et al., 1999] for generating a flux distribution in a volumetric receiver at the PSA CESA plant (figure 3). 


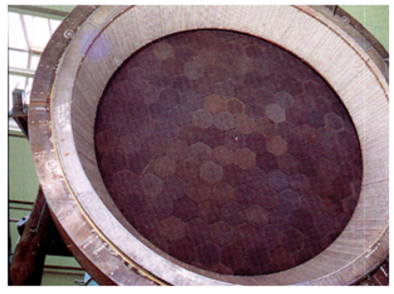

(a) TSA solar receiver

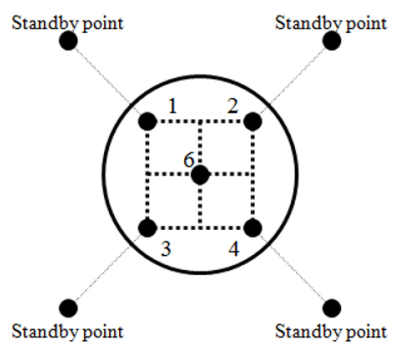

(c) Aimpoints \& standby points

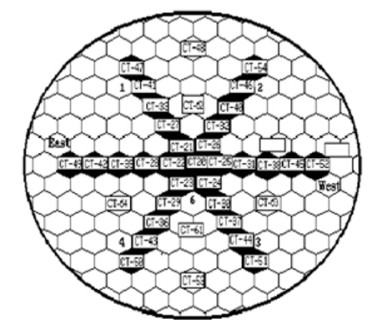

(b) Location of thermocouples

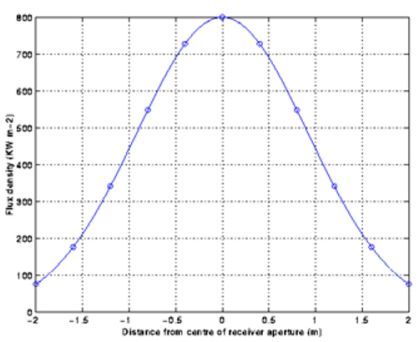

(d) Theoretical flux profile
Fig. 3. Control of the flux conditions

The temperature was measured at the receiver by a series of thermocouples placed in different parts of the receiver. The control algorithm changes the aiming point point coordinates and the heliostats assigned to each of the aiming points to obtain a better distribution of the temperature profile. Figure 4 shows a comparison of the maximum temperature deviation between manual and automatic control operation [García-Martín et al., 1999].

\section{PARABOLIC TROUGH PLANTS}

Parabolic trough systems concentrate sunlight onto a receiver pipe located along the focal line of a trough collector (figure 5). A heat transfer fluid, typically synthetic oil or water, is heated as it flows along the receiver pipe and is routed either to a heat exchanger, when this fluid is oil, to produce steam that feeds an industrial process (for instance a turbine), to a flash tank, when the fluid is pressurized water, to produce steam for an industrial process, or to a turbine when superheated and pressurized steam is produced directly in the solar field [Zarza et al., 2001].

Most of the plants that are operational currently, such as the SEGS plants in California [Price et al., 1990] heat up oil to produce electricity. For a maximum efficiency, a constant supply of hot oil at some prespecified temperature, despite variations in the ambient temperature, the inlet temperature and the direct solar radiation, is required.

During the last 25 years considerable effort has been devoted by many researchers to improve the efficiency of solar thermal power plants with distributed collectors from the control and optimization viewpoints. Most of the work done and summarized in [Camacho et al., 1997] has been devoted to improve the operation of the Acurex field of the SSPS plant located in the PSA, Spain, which uses a parabolic trough systems using oil as heat transfer medium. But there are also some recent experiences controlling parabolic trough systems using water/steam as heat transfer fluid [Zarza et al., 2001, Valenzuela et al., 2004].

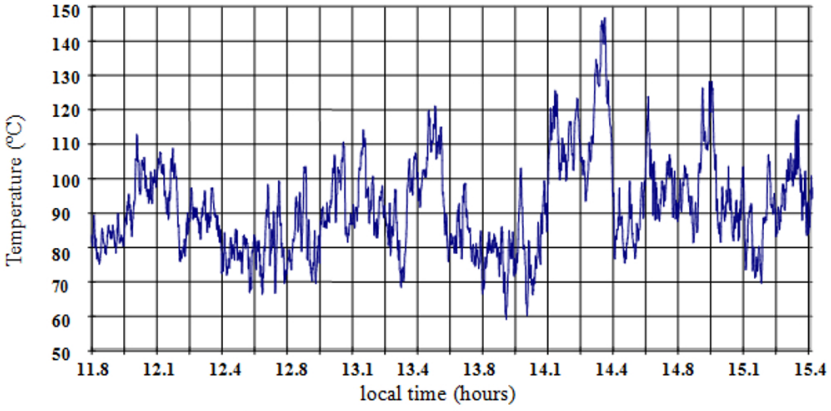

(a) Maximum temperature difference (14/03/97)

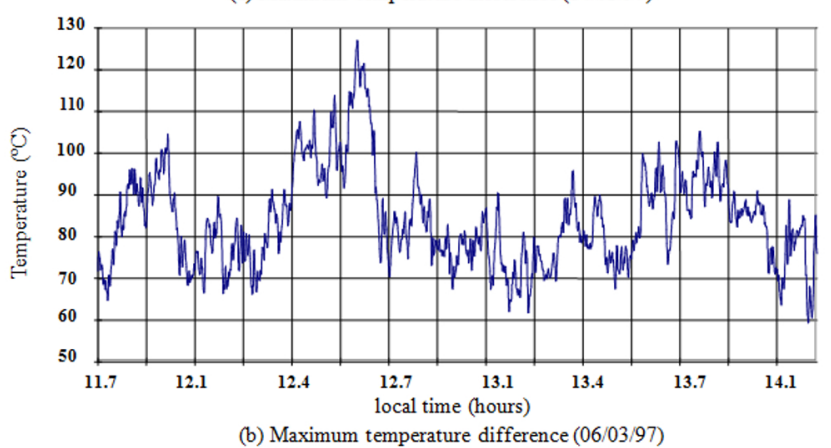

Tests showing the maximum temperature difference within the receiver under automatic operation, [García-Martín et al., 1999]

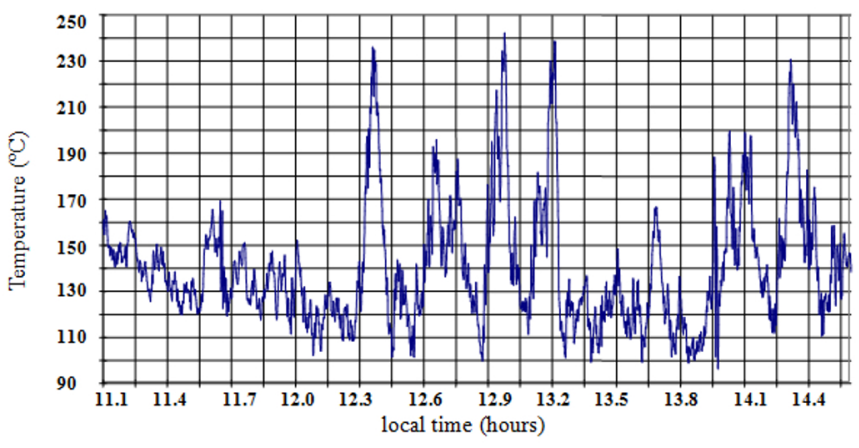

Maximum temperature difference within the receiver under manual operation (07/09/95), [García-Martín et al., 1999]

Fig. 4. Maximum temperature deviation in receiver uniform heating

Currently the SSPS plant is composed of a distributed collector field, a thermal storage system and the power block. The distributed collector field consists of 480 east west aligned single axis tracking collectors forming 10 parallel loops with a total aperture mirrors area of 2672 $\mathrm{m} 2$. Each of the loops is formed by four twelve-module collectors, suitably connected in series. The loop is 172 $\mathrm{m}$ long, the active part of the loop measuring $142 \mathrm{~m}$ and the passive part $30 \mathrm{~m}$. The heat transfer fluid is thermal oil able to support temperatures up to $300^{\circ} \mathrm{C}$, which is pumped from the bottom of a storage tank through the solar field, where picks up the heat transferred through the receiver tube walls, till the top of the tank. The heated oil stored in the tank is used to boil water that is utilized in a steam turbine to drive an electricity generator or to feed a heat exchanger of a desalination plant. The operation limits for the oil pump are between 2.0 and $12.0 \mathrm{l} / \mathrm{s}$ The 


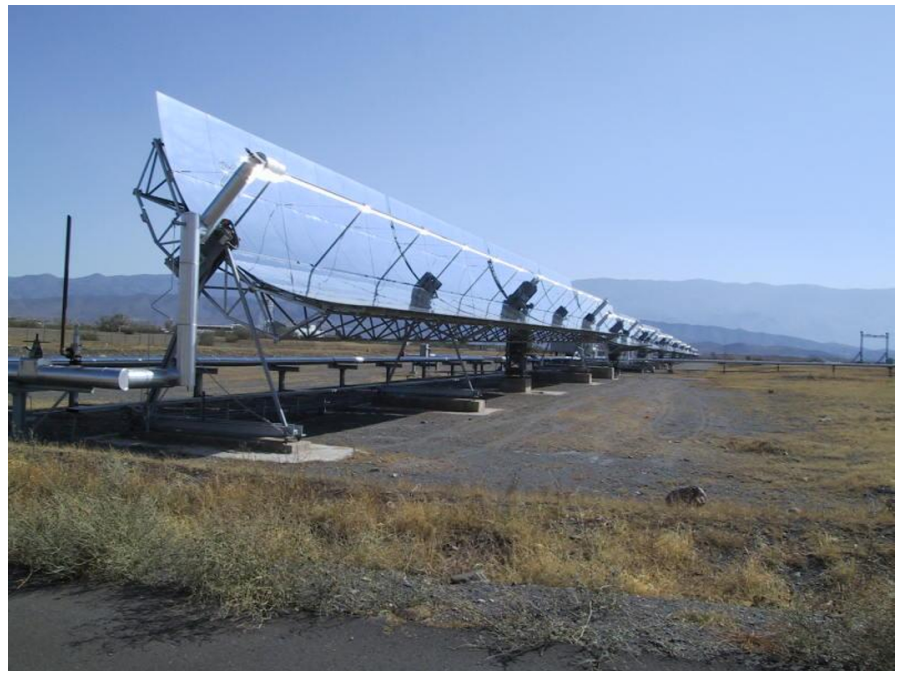

Fig. 5. DISS Parabolic trough at the Plataforma Solar de Almería

minimum value is there for safety and mainly to reduce the risk of the oil being decomposed, which happens when the oil temperature exceeds $305^{\circ} \mathrm{C}$.

The main difference between a conventional power plant and a solar plant is that the primary energy source, while being variable, cannot be manipulated.

The objective of the control system in a distributed collector field is to maintain the outlet oil temperature of the loop at a desired level in spite of disturbances such as changes in the solar irradiance level (caused by clouds), mirror reflectivity or inlet oil temperature. The means available for achieving this is via the adjustment of the fluid flow and the daily solar power cycle characteristics are such that the oil flow has to change substantially during operation. This leads to significant variations in the dynamic characteristics of the field, such as the response rate and the dead time, which cause difficulties in obtaining adequate performance over the operating range with a fixed parameter controller.

The activities performed by the control groups related to this field cover modeling, identification and simulation, classical proportional- integral-derivative control (PID), feedforward control (FF), model based predictive control (MPC), adaptive control (AC), gain-scheduled control (GS), cascade control (CC), internal model control (IMC), time delay compensation (TDC), optimal control (LQG), nonlinear control (NC), robust control (RC), fuzzy logic control (FLC) and neural network controllers (NNC) [Camacho et al., 2007a,b]. An example shown at the end of this paper focuses in the MPC applications. Nonlinear models (both mechanistic and empirical ones) or high order linear models around different operating points have to be used [Arahal et al., 1997]. A distributed solar collector field, under general assumptions and hypotheses, may be described by a distributed parameter model of the temperature [Klein et al., 1974, Rorres et al., 1980, Carmona, 1985, Camacho et al., 1988, Berenguel et al., 1994, Arahal et al., 1997]. The dynamics of the distributed solar collector field are described by the following system of partial differential equations (PDE) describing the energy balance: $\rho_{m} C_{m} A_{m} \frac{\partial T_{m}}{\partial t}=I n_{o} G-G H_{l}\left(T_{m}-T_{a}\right)-L H_{t}\left(T_{m}-T_{f}\right)$

$$
\rho_{f} C_{f} A_{f} \frac{\partial T_{f}}{\partial t}+\rho_{f} C_{f} \dot{q} \frac{\partial T_{f}}{\partial x}=L H_{t}\left(T_{m}-T_{f}\right)
$$

where the subindex $m$ refers to the metal and that of $f$ to the fluid. $H_{l}$ represents the sum of radiative and conductive thermal losses, $\rho_{m}$ and $\rho_{f}$ are the density of the metal and fluid, $C_{m}$ and $C_{m}$ are the heat capacities, $A_{m}$ and $A_{f}$ the tube cross-section and inner area, $I$ is the solar irradiance, $n_{o}$ the reflectivity coefficient, $\dot{q}$ the oil flow, $T_{m}, T_{f}$ and $T_{a}$ are the metal, fluid and ambient temperatures.

The development of numerical simulation models of the plant has played an important role in the design of different control strategies avoiding a number of expensive and time consuming controller tuning tests at the solar power plant. Based on (1) and (2), a distributed parameter model of the Acurex field was developed [Carmona, 1985, Camacho et al., 1988] and implemented [Berenguel et al., 1994, Arahal et al., 1997] and has been used for simulation purposes by many researchers.

Expressions (1) and (2) have also been used for control purposes [Arahal et al., 1997], in the development of feedforward controllers [Camacho et al., 1986, 1992, Berenguel et al., 1994, Meaburn and Hughes, 1997, Johansen et al., 2000], nonlinear PID controllers including a real-time numerical integration of the distributed plant model [Johansen et al., 2000], nonlinear model-based predictive controllers [Camacho and Berenguel, 1994b, Berenguel et al., 1998, Pickhardt and Silva, 1998], internal model control [Farkas and Vajk, 2002], etc.

Most of the MPC strategies applied to the control of solar collectors are in adaptive, robust, or nonlinear fields and including a feedforward term as a part of the controller [Arahal et al., 1997]. Many different MPC strategies [Camacho and Bordóns, 2004] have been applied to control solar collectors.

Few implementations of MPC controllers with fixed parameters have been reported in the literature [Arahal et al., 1997]. The most important applications are: MPC adaptive control [Camacho and Berenguel, 1994a, Camacho et al., 1992, Meaburn and Hughes, 1996, Coito et al., 1997, Rato et al., 1997, Stuetzle et al., 2004], MPC gain scheduling control [Camacho and Berenguel, 1994b, Lemos et al., 2000], MPC robust control [Camacho and Berenguel, 1997, de la Parte et al., 2008] and MPC nonlinear control, including $\mathrm{NNC}$ and feedback linearization (FL) approaches [Camacho and Berenguel, 1994b, Berenguel et al., 1998, Pickhardt and Silva, 1998, Silva et al., 2003].

More recently, a robust MPC controller with tracking capabilities was applied by [Limon et al., 2008a] implementing a previous MPC tracking controller designed in [Limon et al., 2008b]. The plant was assumed to be modelled as a linear system with additive bounded uncertainties on the states.

The remaining part of this section presents a gain scheduling MPC which is one of the most succesful controllers used in the ACUREX plant. Different linear models were 


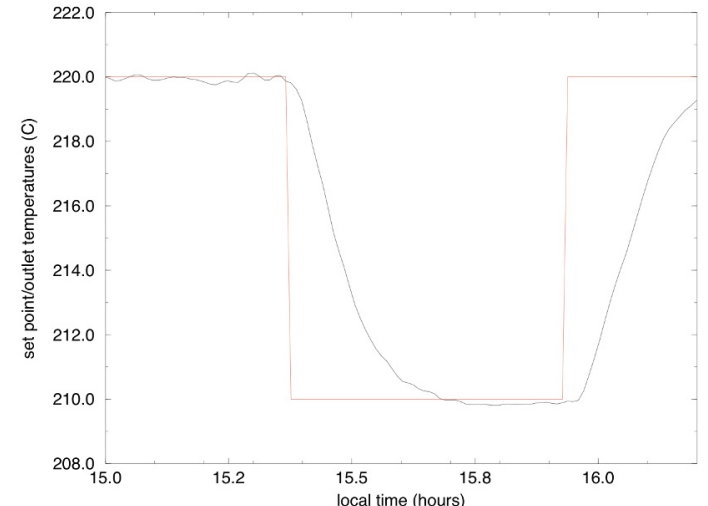

Fig. 6. Set point and oil outlet temperatures ${ }^{\circ} \mathrm{C}$

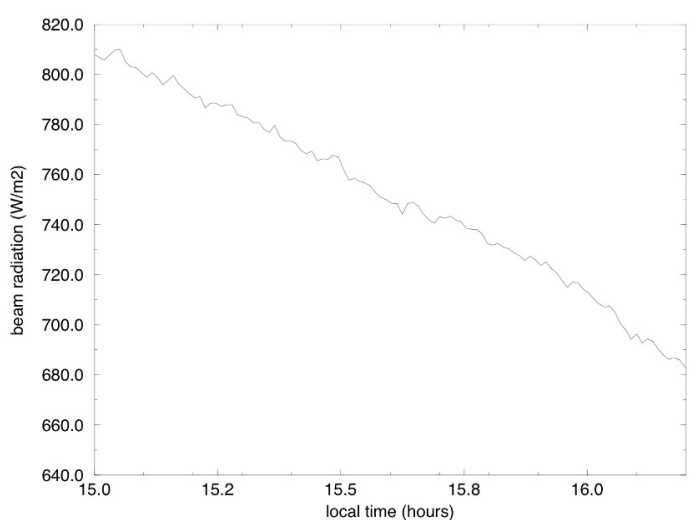

Fig. 7. Solar radiation

obtained from input-output data in different working conditions. These models related changes in oil flow to those of the outlet oil temperature, and can take into account the nonlinearities of the plant if they are adequately adjusted. The output of the MPC is the input of the series compensation controller, which also uses the solar radiation, inlet oil temperature and reflectivity to compute the value of the oil flow which will produce the necessary temperature increment for the given operating conditions and which is sent to the pump controller.

The controller parameters were obtained from a linear model of the plant. From input-output data of the plant, the degrees of the polynomials $A$ and $B$ and the delay (of a CARIMA plant model) that minimizes Akaike's Information Theoretic Criterion (AIC) were found.

Very good results were obtained for different operating point with disturbances due to passing clouds, inlet oil temperature variations and other environmental conditions. Figures 7 and ??. As can be seen, the controller is able to maintain the outlet oil temperature close to the setpoint for different solar radiation conditions.

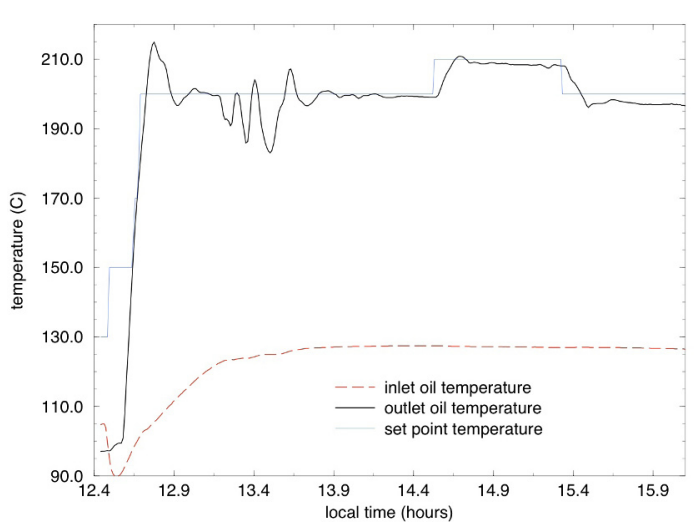

Fig. 8. Set point and oil outlet temperatures ${ }^{\circ} \mathrm{C}$

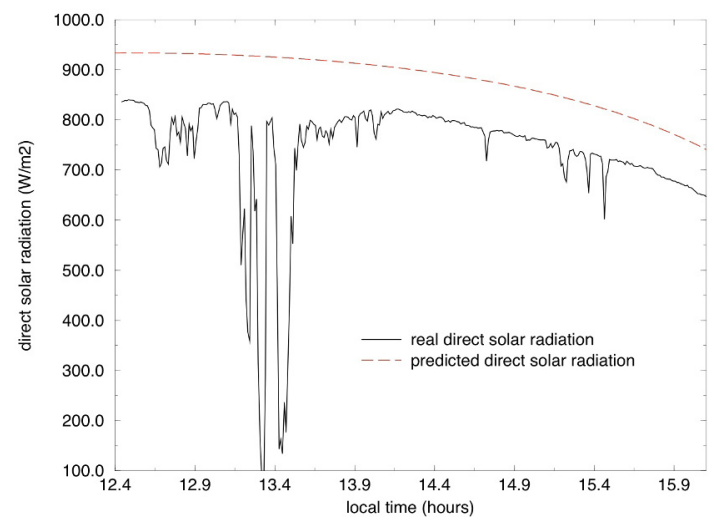

Fig. 9. Solar radiation

Figure 8 shows the outlet oil temperature and the setpoint for a day with strong disturbances in the direct solar radiation because of passing clouds, shown in figure 9 . The setpoint is set at 200 degrees when strong disturbances in the direct solar radiation are produced. As can bee seen, the controller is able to maintain the outlet oil temperature closed to the setpoint inspite of the strong disturbances in the direct solar radiation which changes from $800 \mathrm{~W} / \mathrm{m} 2$ to $100 \mathrm{~W} / \mathrm{m} 2$ in a few seconds.

\section{CONCLUSION}

One of the great technological challenges of our times is to produce solar energy at affordable costs.

Control is one of the enabling technologies to achieve this objective. While in other power generating processes, the main source of energy can be manipulated, in solar energy systems, the main source of power which is solar radiation cannot be manipulated and furthermore it changes in a seasonal and on a daily base acting as a disturbance when considering it from a control point of view. Solar plants have all the characteristics needed for using industrial 
electronics and advanced control strategies able to cope with changing dynamics, nonlinearities and uncertainties. This paper has shown what are the main control problems found in controlling solar power systems and the solutions proposed.

The use of advance control techniques increases the performance of the solar plant because a) advanced control techniques are able to cancel the perturbations caused by passing clouds and this allows the field to be operated under cloudy conditions and b) the technique may dynamically optimize the operational set-points of the plant and maintain the plant closer to the optimal set-points.

The paper has also shown how advanced control techniques can be used to calibrate heliostat and to obtain better flux distribution and thus improving overall plant performance.

\section{ACKNOWLEDGEMENTS}

The authors would like to acknowledge the Spanish Ministry of Science and Innovation and Junta de Andalucía for funding this work under grants DPI2008-05818, DPI201021589-C05-01/04, and P07-TEP-02720

\section{REFERENCES}

Abengoa-Solar. Plataforma solucar: Planta ps20, www.abengoasolar.com/sites/solar, $2009 . \quad$ URL www . abengoasolar.com/sites/solar.

M. R. Arahal, M. Berenguel, and E. F. Camacho. Nonlinear neural model-based predictive control of a solar plant. In Proceedings of the European Control Conference ECC97, July 1997.

M. Berenguel, E. F. Camacho, and F.R. Rubio. Simulation software package for the Acurex field. Dpto. de Ingeniería de Sistemas y Automática, ESII Sevilla, Spain, 1994.

M. Berenguel, M.R. Arahal, and E.F. Camacho. Modeling free response of a solar plant for predictive control. Control Engineering Practice, 6:1257-1266, 1998.

M. Berenguel, F.R. Rubio, A. Valverde, P.J. Lara, M. R. Arahal, E. F. Camacho, and M. Lopez. An artificial vision-based control system for automatic heliostat positioning offset correction in a central receiver solar power plant. Solar Energy, 76-5:523-653, 2004.

M. Blanco, D. Alarcon, T. Lopez, and M. Lara. Computing the solar vector. Solar Energy, 70:431-441, 2001.

B. Bose. Global warming: Energy, environmental pollution, and the impact of power electronics. IEEE Industrial Electronics Magazine, 4(1):6-17, 2010.

E. F. Camacho and M. Berenguel. Aplication of Generalized Predictive Control to a Solar Power Plant. Oxford University Press, Oxford, UK, 1994a.

E. F. Camacho and M. Berenguel. Application of generalized predictive control to a solar power plant. In The Third IEEE Conference on Control Applications, Glasgow UK, August 1994b.

E. F. Camacho and C. Bordóns. Model Predictive Control. Springer-Verlag, second edition, 2004.

E. F. Camacho, R. Carmona, and F.R. Rubio. Adaptive control of the acurex field. In P. Kesserlring and C.S. Selvage, editors, The IEA/SSPS Solar Thermal Power Plants, pages 5.2.1-5.2.18, London, 1986. Springer Verlag.
E. F. Camacho, F.R. Rubio, and J.A. Gutierrez. Modelling and simulation of a solar power plant with a distributed collector system. In Proceedings of the International IFAC Symposium on Power Systems Modeling and Control, 1988.

E. F. Camacho, F.R. Rubio, and F.M. Hugues. Self-tuning control of a solar power plant with a distributed collector field. IEEE Control Systems Magazine, 12:72-78, 1992.

E. F. Camacho, M. Berenguel, and F.R. Rubio. Advanced control of solar power plants. Springer Verlag, London, 1997.

E. F. Camacho, F.R. Rubio, M. Berenguel, and L. Valenzuela. A survey on control schemes for distributed solar collector fields. part i: modeling and basic control approaches. Solar Energy, 81:1240-1251, 2007a.

E. F. Camacho, F.R. Rubio, M. Berenguel, and L. Valenzuela. A survey on control schemes for distributed solar collector fields. part ii: advanced control approaches. Solar Energy, 81:1252-1272, 2007b.

E.F. Camacho and M. Berenguel. Robust Adaptive Model Predictive Control of a Solar Plant with Bounded Uncertainties. International Journal of Adaptive and Signal Processing, 11:311-325, 1997.

E.F. Camacho, M. Berenguel, F.R. Rubio, and D. Martinez. Control of Solar Energy Systems. Springer Verlag, 2012.

R. Carmona. Analysis, Modeling and Control of a Distributed Solar Collector Field with a One-axis Tracking System. PhD thesis, Universidad de Sevilla, 1985.

J.M. Carrasco, L.G. Franquelo, J.T. Bialasiewicz, E. Galvan, R.C.P. Guisado, M.A.M. Prats, J.I. Leon, and N. Moreno-Alfonso. Power-electronic systems for the grid integration of renewable energy sources: A survey. IEEE Transactions on Industrial Electronics, 53(4):1002 $-1016,2006$.

Y. T. Chen, B. H. Lim, and C. S. Lim. Sun tracking formula for heliostats with arbitrarily oriented axes. Journal of Solar Energy Engineering, 128:245-251, 2006.

F. Coito, J.M. Lemos, R.N. Silva, and E. Mosca. Adaptive control of a solar energy plant: exploiting acceptable disturbances. International Journal of Adaptive Control and Signal Processing, 11:327-342, 1997.

M. Perez de la Parte, C.M. Cirre, E. F. Camacho, and M. Berenguel. Application of predictive sliding mode controllers to a solar plant. IEEE Transaction on Control Systems Technology, 16:819-825, 2008.

I. Farkas and I. Vajk. Experiments with internal modelbased controller for acurex field. In Proceedings of the 2nd Users Workshop IHP Programme, CIEMAT, 2002.

F.J. García-Martín, M. Berenguel, and A. Valverde E. F. Camacho. Heuristic knowledge-based heliostat field control for the optimization of the temperature distribution in a volumetric receiver. Solar Energy, 66(5):355-369, 1999

R. Grena. An algorithm for the computation of the solar position. Solar Energy, 82:462-470, 2008.

T.A. Johansen, I. Petersen, and O. Slupphaug. Explicit Suboptimal Linear Quadratic Regulation with input and state constraints. In Proc. 39th IEEE Conf. on Decision and Control, December 2000.

D.L. King and D.E. Arvizu. Heliostat characterization at the central receiver test facility. Journal of Solar Energy Engineering, 103:82-88, 1981. 
A.A. Klein, J.A. Duffie, and W.A. Beckman. Transient considerations of flat-plate solar collectors. Transaction of the ASME Journal of Engineering and Power, 96: 333-344, 1974.

J.M. Lemos, L.M. Rato, and E. Mosca. Integrating predictive and switching control: Basic concepts and an experimental case study. In F. Allgower and A. Zheng, editors, Nonlinear Model Predictive Control, number 1, pages 181-190. 2000.

D. Limon, I. Alvarado, T. Alamo, M.R. Arahal, and E. F. Camacho. Robust control of the distributed solar collector field acurex using mpc for tracking. In $I F A C$ World Congress, 2008a.

D. Limon, I. Alvarado, T. Alamo, and E. F. Camacho. $\mathrm{Mpc}$ for tracking piecewise constant references for constrained linear systems. Automatica, 44(9):2382-2387, 2008b.

D. Limon, I. Alvarado, T. Alamo, and E.F. Camacho. Robust tube-based mpc for tracking of constrained linear systems with additive disturbances. Journal of Process Control, 20:248-260, 2010.

M. Liserre, T. Sauter, and J.Y. Hung. Future energy systems: Integrating renewable energy sources into the smart power grid through industrial electronics. IEEE Industrial Electronics Magazine, 4(1):18-37, 2010.

A. Meaburn and F.M. Hughes. A simple predictive controller for use on large scale arrays of parabolic trough collectors. Solar Energy, 56:583-595, 1996.

A. Meaburn and F.M. Hughes. Feedforward control of solar thermal power plants. Journal of Solar Energy Engineering, 119:52-60, 1997.

R. Pickhardt and R.N. Silva. Application of a nonlinear predictive controller to a solar power plant. In Proceedings of the 1998 IEEE International Conference on Control Applications, 1998.

H. Price, D. Kearney, and I. Replogle. Update on the preformance and operation of segs iii-vii. In $A S M E$ International Solar Energy Conference, 1990.

L. Rato, R.N. Silva, J.M. Lemos, and F. Coito. Multirate musmar cascade control of a distributed solar field. In Proceedings of the European Control Conference ECC97, July 1997.

C. Rorres, A. Orbach, and R. Fischl. Optimal and suboptimal control policies for a solar collector system. IEEE Transactions on Automatic Control, 25: 1085-1091, 1980.

R.N. Silva, L.M. Rato, and J.M. Lemos. Time scaling internal state predictive control of a solar plant. Control Engineering Practice, 11:1459-1467, 2003.

K.W. Stone and C.W. Lopez. Evaluation of the solar one track alignment methodology. Journal of Solar Energy Engineering, 103:521-526, 1995.

T. Stuetzle, N. Blair, J.W. Mitchell, and A. Beckman. Automatic control of a 30mwe segs vi parabolic trough plant. Solar Energy, 76:187-193, 2004.

L. Valenzuela, E. Zarza, M. Berenguel, and E. F. Camacho. Direct steam generation in solar boilers. IEEE Control Systems Magazine, 24(2):15-29, 2004.

E. Zarza, L. Valenzuela, J. Leon, K. Hennecke, M. Eck, H.D. Weyers, and M. Eickhoff. The diss project: Direct steam generation in parabolic troughs. In Proceedings of ASME International Solar Energy, April 2001. 